\title{
Fatores associados à percepção e atitude de consumidores de carne bovina com certificação de origem*
}

\section{Factors associated with perceptions and attitude of consumers of meat with certification of origin}

\author{
Marcos Aurélio Lopes, ${ }^{* *}$ Eline Oliveira Pacheco, ${ }^{* *}$ Fábio Raphael Pascoti Bruhn, ${ }^{* *}$ Fabio Henrique Vicente, ${ }^{* *}$ \\ Peter Bitencourt Faria, ${ }^{* *}$ Christiane Maria Barcellos Magalhães da Rocha**
}

\begin{abstract}
Resumo
O objetivo deste estudo foi verificar quais fatores socioeconômicos estão relacionados com a decisão de compra de carne com certificação de origem, além de levantar o perfil de percepção e atitude dos consumidores de carne bovina em São Luís/MA. Foi realizada a descrição das variáveis e construído um modelo múltiplo Generalized Estimating Equations (GEE) de regressão logística visando a identificar possíveis associações entre as características socioeconômicas dos consumidores e os principais atributos da carne que influenciam a decisão para sua compra levantada por meio das entrevistas entre 400 entrevistados no mês de março de 2012. O selo de qualidade na aquisição da carne foi o atributo que mais influenciou (32,5\%) a decisão de compra dos consumidores. A maioria dos entrevistados $(68,7 \%)$ nunca ouviu falar sobre rastreabilidade bovina. Dentre os que já ouviram falar, a maior parte $(71,2 \%)$ está disposta a pagar mais pela carne com certificação de origem, apesar de considerar que há desvantagens associadas à rastreabilidade, principalmente em relação ao aumento no preço da carne. Consumidores com maior grau de escolaridade apresentam 2,62 mais chances de já terem ouvido falar em carne rastreada e 1,90 mais chances de considerar que o principal benefício da carne rastreada é oferecer mais segurança alimentar; enquanto os com maior renda apresentam 3,23 mais chances de aceitar pagar mais pela certificação da carne. Ou seja, pessoas com maior escolaridade apresentaram melhor conhecimento sobre esse tipo de certificação, enquanto aqueles com maior renda mostraram maior aceitação em pagar mais caro pela carne bovina rastreada.
\end{abstract}

Palavras-chave: atributos da carne, comercialização, rastreabilidade, segurança alimentar.

\begin{abstract}
The aim of this study was to verify which socioeconomic factors are related to the decision to purchase meat with certification of origin, as well as raising the profile of perception and attitude of consumers of meat in São Luís/MA. The description of the variables were performed and constructed a multiple model Generalized Estimating Equations (GEE) of logistic regression model using the statistical package SPSS 18.0, to identify possible associations between socio-demographic characteristics of the consumers and the main attributes of the meat that influence the decision for its purchase raised through interviews among 400 respondents in March 2012. The seal of quality for acquisition of the meat was the attribute that most influences (32,5\%) the purchasing decisions of consumers. Most respondents $(68,7 \%)$ never heard about bovine traceability. Amongst these who had heard about traceability, most $(71,2 \%)$ would be willing to pay more for meat with certification of origin, although considering that there are disadvantages associated with traceability, especially in relation to the increase in the price of meat. Consumers with greater levels of schooling present 2,62 more chances of having already heard of traced meat and 1,90 more chances of considering that the main benefit of traced meat is to offer more food security, while the ones with bigger income present 3,23 more chances of accepting to pay more for the certification of the meat. This means that people with greater schooling present better knowledge about this type of certification, while those with bigger income show bigger acceptance to spend more money on traced meat.
\end{abstract}

Keywords: Attributes of meat, marketing, traceability, food safety

\section{Introdução}

A segurança alimentar desempenha um papel estratégico no comércio mundial e representa uma preocupação global (Nesbaken, 2009). De acordo com Sofos (2008), os desafios relacionados com a segurança de produtos de origem animal e as dificuldades no controle eficiente dos processos geram a necessidade de criar mecanismos que reduzam o risco potencial de doenças transmitidas por alimentos.
Neste sentido, destaca-se a rastreabilidade como meio de integração de toda a cadeia produtiva em um sistema de monitoramento e certificação, melhorando a percepção de segurança para os consumidores devido à geração de maior número de informações sobre a carne (Verbeke et al., 2010). Sendo o Brasil um dos maiores fornecedores de carne bovina para União Europeia, uma das exigências para continuar com as exportações foi a implantação do programa de rastreabilidade.

${ }^{*}$ Recebido em 28 de abril de 2014 e aceito em 21 de junho de 2014.

**Departamento de Medicina Veterinária(DMV), Universidade Federal de Lavras (UFLA), Cx. Postal 3037, Lavras, MG, Brasil. CEP $37200-000$.

Autor para correspondência: malopes@dmv.ufla.br 
A rastreabilidade é a identificação da origem do produto, processado ou não, até o seu consumidor por meio de medidas que ajudam no controle e monitoramento de todas as entradas e saídas, garantindo a qualidade da produção (lba et al., 2003). Segundo Ubilava e Foster (2009), a certificação de qualidade é uma segmentação de mercado que leva a oportunidades para processadores de alimentos e varejistas, por serem considerados parte do sistema de gestão e envolver controle do sistema de produção.

Nesse contexto, os pontos de venda podem comercializar produtos classificados como carne rastreada ou apenas com certificação de origem. A carne rastreada difere da com certificação de origem, por possuir outros dados importantes além da origem do animal, como o registro de diversas ocorrências na vida do animal, datas e tipos de vacinas, tratamentos veterinários associados, indicações do período de carência dos medicamentos administrados, transferências de propriedades e rotas trânsito do animal, bem como sexo e aptidão (Latouche et al., 1998; Lopes et al., 2007).

O sistema de rastreabilidade busca melhorar a segurança nos processos de obtenção da carne e dos seus produtos. Entretanto, estes benefícios não são tão claros para os consumidores como são para os pesquisadores e indústria (Wezemael et al., 2011).

Torna-se relevante, portanto, avaliar se os consumidores conhecem o conceito de rastreabilidade bovina e se a carne com certificação de origem, que atualmente é comercializada nas redes de supermercados, está incluída no desejo de compra dos mesmos, ainda que se pague mais caro por esse produto. Assim, os objetivos desta pesquisa foram verificar a associação entre os aspectos socioeconômicos dos consumidores e os principais atributos da carne que influenciam a decisão para sua compra, assim como o conhecimento sobre rastreabilidade bovina $e$ disposição para pagar a mais pela carne com certificação de origem, além de levantar o perfil de percepção e atitude dos consumidores de carne bovina rastreada em São Luis/MA.

\section{Material e métodos}

Foi realizado um estudo observacional de corte transversal em dez supermercados na cidade de São Luís, capital do estado do Maranhão (MA), Brasil, em março de 2012, para avaliar a associação entre a percepção e atitude de consumidores de carne bovina rastreada e algumas características sociodemográficas. A definição do número de entrevistados $(n=400)$ necessário baseou-se em Barbetta (2007), considerando um erro amostral máximo de $5 \%$ e uma população de 1.014 .837 mil habitantes de São Luís (IBGE, 2010).

Visando obter informações sobre a caracterização da percepção e atitude de consumidores de carne bovina, foram realizadas entrevistas, por um único pesquisador, a partir de formulário estruturado (36 perguntas fechadas) (Tabela 1), adaptados de Velho et al. (2009). Ainda, para todos os entrevistados foram obtidas informações sobre algumas características sociodemográficas relacionadas com o sexo, idade (até 40 anos; mais que 40 anos), escolaridade (até segundo grau; graduação ou pós-graduação) e renda (até seis salários mínimos; mais que seis salários mínimos) utilizadas como variáveis independentes no estudo. Os entrevistados foram selecionados aleatoriamente, sendo convidados enquanto se encontravam em frente à gôndola de carnes no supermercado, sendo que para os consumidores
Tabela 1: Resumo das características levantadas em entrevistas aplicadas a consumidores de carne bovina em supermercados de São Luís/MA, Brasil, 2012

\begin{tabular}{|c|c|}
\hline Item & Variáveis dicotômicas (Respostas: não/sim) \\
\hline \multirow{3}{*}{ Atitude } & $\begin{array}{l}\text { Consumo de produtos de origem animal com certifi- } \\
\text { cação }\end{array}$ \\
\hline & $\begin{array}{l}\text { Disposição em pagar mais caro pelo produto de ori- } \\
\text { gem animal certificado }\end{array}$ \\
\hline & $\begin{array}{l}\text { Disposição em pagar a mais pela carne rastreada: } 1 \text {. } \\
\text { até } 5 \% \text {; } 2.5 \% \text {; 3. } 10 \% \text {; } 4.15 \% ; 5.20 \% \text {; ou } 6 \text {. mais } \\
\text { que } 20 \%\end{array}$ \\
\hline \multirow{5}{*}{ Percepção } & $\begin{array}{l}\text { Se já tinha ouvido falar sobre rastreabilidade, carne } \\
\text { rastreada ou carne com garantia de origem }\end{array}$ \\
\hline & $\begin{array}{l}\text { Atributo mais importante na decisão de compra da } \\
\text { carne: } 1 \text {. presença do selo de qualidade; } 2 \text {. carimbo } \\
\text { do SIF; } 3 \text {. preço; } 4 \text {. quantidade de gordura; } 5 \text {. preparo } \\
\text { rápido/fácil; } 6 \text {. cria ou engorda; } 7 \text {. local de abate; e } 8 \text {. } \\
\text { Local de compra; } 9 \text {. raça; e } 10 \text {. sexo do animal; } 11 . \\
\text { maciez/textura; } 12 \text {. odor; e 13. Cor da carne }\end{array}$ \\
\hline & $\begin{array}{l}\text { Conceito de carne rastreada: } 1 \text {. Carne que traz as } \\
\text { informações nutricionais na embalagem; } 2 \text {. Produto } \\
\text { cárneo que não possui contaminantes ou resíduos } \\
\text { químicos; } 3 \text {. Carne que traz as informações relacio- } \\
\text { nadas ao sistema de produção e origem do animal; e } \\
4 \text {. Carne com fiscalização pelo sistema de inspeção } \\
\text { federal ou serviço estadual }\end{array}$ \\
\hline & $\begin{array}{l}\text { Benefícios que a carne rastreada poderia superar a } \\
\text { sem rastreabilidade: } 1 \text {. maior competitividade no mer- } \\
\text { cado exterior, } 2 \text {. melhor qualidade, mais saborosos e } \\
\text { nutritivos; 3. maior segurança e menor risco a saúde } \\
\text { pública; } 4 \text {. acesso a informações referentes à idade, } \\
\text { sexo, raça e outras; e } 5 \text {. nenhum }\end{array}$ \\
\hline & $\begin{array}{l}\text { Desvantagens que a carne com rastreada poderia } \\
\text { trazer: } 1 \text {. maior preço; } 2 \text {. menor oferta; } 3 \text {. valorização } \\
\text { de todas as carnes; e } 4 \text {. nenhuma }\end{array}$ \\
\hline
\end{tabular}

que nunca haviam ouvido falar sobre carne bovina rastreada, a entrevista se restringia às questões relativas às características sociodemográficas.

Para identificar as características sociodemográficas associadas à percepção e atitude dos consumidores em relação à carne bovina com certificação de origem, foi realizada a análise bivariada por teste qui-quadrado $\left(x^{2}\right)$ ou Exato de Fischer; este último, quando constatou-se menos que cinco observações no quadrante do teste. As variáveis que mostraram associação com $\mathrm{P} \leq 0,2$ pelo teste de $x^{2}$ ou Exato de Fischer foram selecionadas para a construção do modelo múltiplo.

Assim, a existência de associação entre os fatores foi verificada utilizando-se o modelo múltiplo Generalized Estimating Equations (GEE) de regressão logística, que é mais adequada quando se observam dados associados em diferentes dimensões (Hanley et al., 2003), como neste estudo, em que se consideram as respostas de consumidores presentes em diferentes supermercados (considerados sujeitos do modelo). A análise GEE permite a avaliação de associação de múltiplos indivíduos considerando o supermercado em que compravam no modelo, assumindo que os casos são dependentes dentro de cada sujeito e independentes entre sujeitos. Para todas as 
variáveis presentes no modelo final $(p<0,05)$, foi estimado o risco por meio da Odds Ratio (OR) ajustada e seu intervalo de confiança de $95 \%$.

\section{Resultados}

Neste estudo, a maior parte dos entrevistados foi do sexo feminino $(53,3 \%)$, com até 40 anos $(63,8 \%)$, apresentava até segundo grau de escolaridade $(57,8 \%)$ e renda de até seis salários mínimos (82,7\%). Deste total, $31,2 \%$ relataram já terem ouvido falar em carne rastreada e $60,7 \%$ alegaram consumir produtos de origem animal com certificação (Tabela 2).

Tabela 2: Características sociodemográficas dos consumidores de carne de São Luís entrevistados $(n=400)$

\begin{tabular}{ccc}
\hline Variável & Categoria & $\%$ \\
\hline Sexo & Masculino & 46.8 \\
& Feminino & 53.3 \\
Idade & Até 30 anos & 39.0 \\
& Até 40 anos & 63.8 \\
& Até 60 anos & 96.3 \\
Escolaridade & Até $1^{\circ}$ grau & 11.1 \\
& Até ${ }^{\circ}$ grau & 57.8 \\
Renda* & Até $3^{\circ}$ grau & 90.2 \\
& Até 3 salários mínimos & 57.9 \\
& Até 6 salários mínimos & 82.7 \\
& Até 10 salários mínimos & 91.8 \\
Já ouviram falar em & Sim & 31.3 \\
rastreabilidade & Não & 68.7 \\
\hline
\end{tabular}

* salário mínimo=R $\$ 622,00$

Dentre os consumidores que já ouviram falar em carne rastreada, o principal critério considerado na decisão de compra da carne bovina foi: a presença do selo de qualidade $(32,5 \%)$, presença do carimbo do Serviço de Inspeção Federal (SIF) ou estadual $(17,3 \%)$, cheiro $(15,7 \%)$, preço $(10,5 \%)$, cor $(10,4 \%)$, menor teor de gordura $(7,3 \%)$, local de abate $(4,2 \%)$ e estabelecimento comercial onde foi realizada a aquisição da carne $(2,1 \%)$. Alem disso, $36,3 \%$ citaram o correto conceito de carne rastreada "produto que traz as informações de origem".

Os maiores benefícios considerados pelos consumidores foram: maior segurança alimentar e menor risco para a saúde pública $(56,0 \%)$, produtos com melhor qualidade, mais saborosos $e$ nutritivos $(26,4 \%)$. Grande parte dos entrevistados $(65,6 \%)$ considera que há desvantagens da carne rastreada em relação à sem rastreabilidade, sendo o aumento no preço do produto a principal alegação $(52,0 \%)$. Apesar disso, a maior parte relatou que aceitaria pagar mais caro pela carne rastreada $(71,2 \%)$, somente um acréscimo de até $5 \%(41,6 \%)$.

Neste estudo foi observado que indivíduos do sexo feminino têm menos chance $(p=0,020 ; O R=0,539 ; I C=95 \%=1,072-3,339)$ de já terem ouvido falar em carne rastreada, e que pessoas com escolaridade maior que o $2^{\circ}$ grau apresentam mais chance $(p=0,007 ; O R=2,625 ; \mathrm{IC}=95 \%=1,307-5,270)$ de já terem ouvido falar em carne rastreada. Observou-se também que indivíduos do sexo feminino apresentam menos chance $(p=0,011 ; O R=0,479$; $\mathrm{IC}=95 \%=0,272-0,845$ ), enquanto os com escolaridade maior que $\circ 2^{\circ}$ grau apresentam mais chance $(p=0,001$; $O R=2,588$; IC=95\%=1,463-4,578) de considerar o carimbo do SIF como um dos atributos mais importantes na compra de carne. Além disso, pessoas com escolaridade maior que $\circ 2^{\circ}$ grau apresentam menos chance $(p=0,012 ; O R=0,402 ; \mathrm{IC}=95 \%=0,197-0,821)$ de considerar a cor como um dos atributos mais importantes na compra de carne e mais chance $(p=0,020$; OR= 1,909 ; $I C=95 \%=1,106-3,297)$ de considerar que os benefícios da carne rastreada em relação à carne sem rastreabilidade é oferecer mais segurança e evitar riscos de doenças.

Em relação à aceitação em pagar mais caro pela rastreabilidade, entrevistados com mais de 40 anos apresentam mais chance ( $p=$ $0,028 ; \mathrm{OR}=1,892 ; \mathrm{IC}=95 \%=1,072-3,339)$ de aceitar pagar mais caro pelo produto de origem animal, e ainda pessoas com renda maior do que seis salários mínimos apresentam mais chance $(p=0,000 ; O R=3,231 ; \mathrm{IC}=95 \%=1,807-5,777)$ de aceitar pagar até $20 \%$ a mais pela certificação da carne.

\section{Discussão}

Apesar de não observado no presente estudo, uma vez que o custo do produto foi o quarto fator mais citado (citado por $10,5 \%$ dos entrevistados), o atributo preço é geralmente o fator considerado de maior impacto na decisão da compra da carne pelo consumidor (Verbeke \& Vackier, 2004; Hocquette et al., 2012). Angulo \& Gil (2007), em estudo realizado na Espanha, relataram que o preço é um dos fatores mais importantes na percepção do risco de segurança alimentar e que os consumidores acreditam que produtos mais caros apresentam algum tipo de vantagem. Semelhante aos resultados desse trabalho, Tonsor \& Marsh (2007), verificaram nos Estados Unidos que aproximadamente $75 \%$ da variabilidade na demanda ou de consumo está relacionada com fatores além do preço da carne e renda.

No presente estudo, os consumidores apresentaram uma maior tendência em confiar mais no sistema de inspeção federal ou estadual, baseado no selo de qualidade e no carimbo do SIF, como modelo de garantir a qualidade da carne comprada, sendo estes os fatores mais citados como decisivos no momento de escolha da carne (citado por $49,8 \%$ dos entrevistados). Neste sentido, segundo Unnevehr et al. (2010), questões éticas e sociais são variáveis que apresentam grande influência sobre a escolha de alimentos principalmente em países desenvolvidos. Em um levantamento com consumidores de carne bovina de cinco países europeus (França, Alemanha, Polônia, Espanha e Reino Unido), Wezemael et al. (2011) verificaram que o maior nivel de confiança e a percepção de risco para a carne bovina estavam associados a uma melhor aceitação dos consumidores para o uso de técnicas que melhorassem a sua segurança, principalmente em relação ao seu processo de obtenção, como no caso da adoção da rastreabilidade no sistema produtivo. Porém Krystallis et al. (2007), na Grécia, verificaram que os consumidores deste país preferem realizar a compra da carne diretamente em açougues, onde acreditam poderem ter certeza de sua origem, em vez de consultarem as informações disponíveis nos rótulos dos produtos encontrados em supermercados, ou seja, preferem confiar na qualidade visual do que em marcas e certificações de qualidade. 
No presente estudo, grande parte dos entrevistados $(26,1 \%)$ relataram que as características sensoriais externas (cor e cheiro) são as principais a serem observadas na decisão de compra. Neste sentido, De Zen e Brandão (1998), em estudo feito na cidade de São Paulo, identificaram que os consumidores de carne bovina, de maneira geral, também valorizam a qualidade do produto por características externas, mas que essa valorização, para as classes com renda mais baixa, perde a importância para o preço do produto. Já a quantidade de gordura foi relatada como sendo um fator menos importante, citado por apenas 7,3\% dos entrevistados. Entretanto, trabalho realizado por Brisola e Castro (2005) com 413 consumidores do Distrito Federal mostrou que $76,4 \%$ dos entrevistados dão muita importância à quantidade de gordura presente na carne.

Por outro lado, alguns autores citam que o consumidor realiza a sua decisão de compra da carne baseando-se em um grande número de informações (preço, etiqueta, marca, aparência e tipo de corte), que por sua vez estariam relacionados, na sua concepção de qualidade, em termos dos atributos maciez, sabor, frescor e nutrição (Krystalliset al., 2007; Verbeke et al., 2010; Troy \& Kerry, 2010). Contudo, esse comportamento está mudando, assim como verificado no presente estudo, uma vez que a maior parte dos entrevistados relatou que observam a presença dos carimbos de inspeção oficiais no momento da compra da carne, portanto, têm voltado uma maior atenção à segurança alimentar, principalmente relacionado com características extrínsecas dos produtos, tais como o sistema de produção animal como a utilização de tecnologias, produção orgânica, técnicas de biotecnologia, uso de antibióticos, pesticidas e hormônios de crescimento (Unnevehr et al., 2010). Segundo Sato \& Silva (2008), o perfil do consumidor está mudando devido ao maior acesso à informação; esses estão cada vez mais exigentes em relação aos atributos de qualidade da carne.

Os resultados sobre o conhecimento a respeito de rastreabilidade verificados nesta pesquisa, na qual grande parte dos consumidores $(36,3 \%)$ citou o correto conceito de carne rastreada - "produto que traz as informações de origem" foram maiores que os encontrados por Zhao et al. (2010), no qual apenas $3 \%$ dos 588 entrevistados nas cidades de Beijing e Xianyang, na China, estavam familiarizados com o real conceito de carne rastreada. Um dos fatores que contribuiu para o baixo índice de familiaridade na China, segundo esses autores, é o fato de que a rastreabilidade é recente nesse país, sendo as primeiras importações realizadas em 2009.

Somente $6,25 \%$ dos entrevistados consideraram que não há benefício da carne rastreada em relação à não rastreada, sendo o benefício mais citado maior segurança alimentar e menor risco para a saúde pública (56,0\%). Morkbak et al. (2008) apontaram que os consumidores apresentam uma disposição positiva à compra do produto quando, dentre outros atributos, existe a garantia de que ele é seguro. Segundo Grunert et al. (2004), é necessário assegurar uma garantia de origem do produto, mostrando que o mesmo foi produzido dentro de boas práticas de fabricação e que está apto a receber seu certificado de origem, analogicamente ao certificado de garantia de qualquer outro produto, monstrando confiança para a sua compra. Para aprimorar a percepção de segurança alimentar, Angulo e Gil (2007) salientaram que os sistemas de controle da qualidade devem ser fortalecidos e divulgados para o maior conhecimento da população.
Grande parte dos entrevistados considera que há desvantagens da carne rastreada em relação à sem rastreabilidade, sendo o aumento no preço do produto a principal alegação $(52,0 \%)$. Apesar disso, a maior parte relatou que aceitaria pagar mais caro pela carne rastreada $(71,2 \%)$, somente acréscimo de até $5 \%(41,6 \%)$, o que pode ser considerado um valor baixo, uma vez que de acordo com Lazzarotto (2011), no Brasil a carne certificada pode apresentar valores até $30 \%$ maiores que o produto que não apresenta certificação de origem. Zhao et al. (2010) encontraram percentuais superiores aos citados no presente estudo, relatando que a maioria dos entrevistados na China pagariam de 9 a $12 \%$ a mais por produtos rastreados.

$\mathrm{Na}$ Coréia, onde grande parte da carne consumida é importada, Lee et al. (2011), verificaram uma disposição dos consumidores em pagar até 39\% para a carne bovina rastreada em relação à não rastreada. Esse resultado, segundo os referidos autores, reflete a preocupação dos consumidores com a segurança alimentar em relação à carne importada dos Estados Unidos, devido à ocorrência da Encefalopatia Espongiforme Bovina (EEB) em anos anteriores. Resende Filho (2008) avaliando potenciais benefícios da rastreabilidade animal para o setor de carnes americano, estimou que o custo para a implantação do sistema seria pago com o ganho em receita, com uma estimativa entre o custo e a receita em torno de $30 \%$. Pendell et al. (2010) realizaram, nos Estados Unidos, uma avaliação do impacto sobre o custo de produção para utilização da identificação e rastreamento, citando que estes representariam os seguintes percentuais de aumento: valor de $0,007 \%$ no preço da carne no atacado; $0,12 \%$ em nível de abate de bovinos e, de $0,43 \%$ em nível de fazenda.

Em relação aos custos com a rastreabilidade, segundo alguns autores, estes são passíveis de serem suportados pela indústria em decorrência de uma maior demanda do produto devido ao aumento da confiança dos consumidores em relação à carne bovina (Resende Filho, 2008; Pendell et al., 2010). Apesar disso, os resultados deste trabalho demostram que a percepção dos consumidores de carne no estado do Maranhão é inadequada, uma vez que a maior parte desconhece o termo rastreabilidade, e dentre os que o conhecem, a maior parte não soube citar o conceito correto de carne rastreada. Também foi observado que essa percepção reflete na atitude dos consumidores, uma vez que a maior parte não aceitaria um acrescimo maior de $5 \%$ nos preços dos produtos rastreados. Esse comportamento pode ser em função da não ocorrência de casos da doença (BSE) e pela posição do país como grande exportador de carne.

Neste estudo foi observado que, no estado do Maranhão, o sexo dos entrevistados interfere no conhecimento de carne rastreada, já que mulheres apresentam menores chances de já terem ouvido falar nessa variável e de considerar o carimbo do SIF como fator mais importante na decisão de compra da carne.

No presente estudo, os entrevistados que possuíam maior nível de escolaridade apresentaram mais chances de considerar que um dos benefícios da carne rastreada, em relação à não rastreada, está relacionado com o produto oferecer maior segurança em relação a riscos de transmissão de doenças. Esses entrevistados também apresentaram maior conhecimento sobre o termo rastreabilidade e consideraram a presença do carimbo do SIF como fator mais importante para a decisão de compra da carne. Esses resultados indicam que a escolaridade 
contribui para aumentar a conscientização das pessoas em relação à importância da carne rastreada, devido ao maior acesso às informações de sua obtenção, proporcionando uma maior transparência e compreensão das etapas envolvidas no processo produtivo, o que acarreta um maior nível de garantia e segurança ao produto final.

Outro aspecto que reflete de forma positiva sobre ao aumento do conhecimento sobre a rastreabilidade, são os consumidores de carne que possuem filhos. Segundo Verbeke \& Vackier (2004), este público demonstra maior cautela na tomada de decisão no momento da compra de carne, buscando informações a respeito da origem e características dos produtos, com maior preocupação em relação à segurança alimentar e uma dieta saudável.

Neste estudo também foi observado que indivíduos com mais de 40 anos e maior renda mensal apresentam maior probabilidade de aceitar pagar mais caro pela carne com certificação de origem. Ou seja, maiores níveis de renda e idade são fatores de influência sobre a aceitabilidade dos consumidores em pagar mais caro pela carne bovina rastreada, como era esperado, o que provavelmente está associado à melhor percepção destes indivíduos em relação aos benefícios deste tipo de produto.

Considerando o perfil dos consumidores de carne bovina em seus diferentes aspectos, para proporcionar maior difusão

\section{Referências}

ÂNGULO, A.M.; GIL, A.J.M. Risk Perception and consumer willingness to pay for certified beef in Spain. Food Qual. Prefer, v.18, p. 1106-1117, 2007.

BARBETTA, P.A. Estatística aplicada às Ciências Sociais. $7 \cdot$ ed. Florianópolis: UFSC, 2007. $315 \mathrm{p}$

BRISOLA, M.V.; CASTRO, A.M.G. Preferências do consumidor de carne bovina do Distrito Federal pelo ponto de compra e pelo produto adquirido. Caderno de Pesquisas em Administração, São Paulo, v. 12, n. 1, p. 81-99, 2005.

De ZEN, S.; BRANDÃO, M.M. Perfil do consumidor de carne bovina. Preços Agri., v.12, p. 3-7, 1998.

GRUNERT, K.G.; BRUNSO, K.; BREDAHL, L.Consumer perception of meat quality and implications for product development in the meat sector - a review. Meat Sci., v. 66, p. 259-272, 2004.

HANLEY, A.J.; NEGASSA, A.; EDWARDES, M.D.B.; FORRESTER, J.E. Statistical Analysis of Correlated Data Using Generalized Estimating Equations: An Orientation. Am. J. Epidemiol., v. 157, p. 364-375, 2003.

HOCQUETTE, J.F.;BOTREAU R.; PICARD, B.; JACQUET, A.;PETHICK, D.W.; SCOLLAN, N.D. Opportunities for predicting and manipulating beef quality. Meat Sci., v. 92, p.197-209, 2012

IBA, S.K.; BRABET C.; OLIVEIRA I.J.; PALLET, D. Um panorama da rastreabilidade dos produtos agropecuários do Brasil destinados à exportação - carnes, soja e frutas. Escola Superior de Agricultura Luiz de Queiroz/Centre International de Recherche Agronomique pour le développement, 2003. Disponível em: <http://www.cendotec.org.br/dossier/cirad/produitsbrpr.pdf>. Acesso em: 14 abril, 2013.

IBGE. Instituto Brasileiro de Geografia e Estatística: Cidades, 2010. Disponível em: <http://www.ibge.gov.br/cidadesat/ topwindow.htm?1>. Acesso em: 23 fev, 2014. e demanda para os produtos com certificação de origem ou rastreados, é necessária a adoção de estratégias de veiculação de informações de forma mais direta, principalmente a partir do local onde adquirem os produtos, como redes de supermercados e casas de carnes especializadas, pois o conhecimento se mostrou o fator mais importante na decisão e atitude relacionados com o consumo de carne bovina.

\section{Conclusões}

O nível de escolaridade e sexo influenciam o conhecimento e avaliação de parâmetros no momento da aquisição de carne bovina, sendo que a maioria das mulheres e entrevistados com nível de escolaridade abaixo do $2^{\circ} \mathrm{Grau}$ desconhecem os conceitos de rastreabilidade e a importância de se adquirir produtos com selo de inspeção.

Entrevistados que possuem nível de escolaridade acima do $2^{\circ}$ grau, que tem mais de 40 anos e renda superior a 6 salários mínimos relacionam a rastreabilidade da carne bovina como um fator ligado à maior segurança alimentar e estão dispostos a pagar até $20 \%$ a mais no preço do produto. Tal fato indica que esse público apresenta melhor percepção em relação aos aspectos de qualidade de carne e busca maiores informações a respeito dos produtos que estão adquirindo.

KRYSTALLIS, A.; CHRYSSOCHOIDIS, G.; SCHOLDERER, J. Consumer-perceived quality in 'traditional' food chains: The case of the Greek meat supply chain. Appet., v. 48, p. 54-68, 2007.

LATOUCHE, K.; RAINELLI P.; VERMERSCH, D. Food safety issues and the BSE scare: some lessons from the French case. Food Pol., v. 23, p. 347-356, 1998.

LAZZAROTTO,N.F. Estudos sobre o mercado de certificações em alimentos no Brasil, 2011. Disponível em: <http://www.fearp.usp. br/ egna/resumos/Lazzarotto.pdf> Acesso em: 21 jun, 2011.

LEE, J.Y.; HAN, D.B.; NAYGA JUNIOR, R.M.; LIM, S.S. Valuing traceability of imported beef in Korea: an experimental auction approach. The Aust. J. Agri. Res. Econ., v. 55, p. 360-373, 2011.

LOPES, M.A.; SANTOS ,G.; AMADO, G.B.; CARDOSO, M.G. Principais dificuldades encontradas pelos pecuaristas na implantação da rastreabilidade de bovinos. Cienc. An. Bras., v. 8, p. 515-520, 2007.

MØRKBAK, M.R.; CHRISTENSEN T.; GYRD-HANSEN D. Valuation of food safety in meat e a review of stated preference studies. Food Econ., v. 5, p. 63-74, 2008.

NESBAKEN, T. Food Safety in a global market - Do we need to worry? Small Rum. Res., v. 86, p. 63-66, 2009.

PENDELL, D.L.; BRESTER, G.W.; SCHROEDER, T.C.; DHUYVETTER, K.C.; TONSOR, G.T. Animal identification and tracing in the united states. Amer. J. Agr. Econ., v. 92, p. 927940, 2010.

RESENDE FILHO, M.A. Potenciais benefícios do sistema de rastreabilidade animal dos EUA para o setor de carnes americano. Rev. Econ. Sociol. Rural, v. 46, p.1129-1154, 2008.

SATO, G.S.; SILVA, R.O.P. Fragilidade do modelo de rastreabilidade da carne bovina brasileira. Analises Indicadores. Agron., v. 3, p. 1-5, 1991.

SOFOS, J.N. Challenges to meat safety in the 21 st century. Meat Sci., v. 78, p. 3-13, 2008. 
TROY, D.J.; KERRY, J.P.Consumer perception and the role of science in the meat industry. Meat Sci.,v. 86, p. 214-226, 2010.

TONSOR, G.; MARSH, G.L.Comparing Heterogeneous Consumption in U.S. and Japanese Meat Fish Demand. Agri. Econ., v. 37, p. 81-91, 2007.

UBILAVA, D.; FOSTER ,K. Quality certification vs. product traceability: Consumer preferences for informational attributes of pork in Georgia. Food Pol., v. 34, p. 305-310, 2009.

UNNEVEHR ,L.; EALES, J.; JENSEN ,H.; LUSK, J.; MCCLUSKEY, J.; KINSEY, J. Food and Consumer Economics. Am. J. Agri. Econ., v. 92, p. 506-521, 2010.

VELHO, J.P.;BARCELLOS, J.O.J.;LENGLER, L.;ELIAS, S.A., OLIVEIRA,T.E. Disposição dos consumidores porto-alegrenses à compra de carne bovina com certificação. Rev. Bras. Zootec., v. 38, p. 399-404, 2009.
VERBEKE, W.; VACKIER, I. Profile and effects of consumer involvement in fresh meat. Meat Sci., v. 67, p. 159-168, 2004. VERBEKE, W.; WEZEMAEL, L.V.; BARCELLOS, M.D.; KUGLER, J.O.; HOCQUETTE, J.F.; UELAND, O.; GRUNERT, K.G. European beef consumer's interest in a beef eating-quality guarantee: insights from a qualitative study in four EU countries. Appet., v. 54, p. 289-296, 2010.

WEZEMAEL, L.V.; WERBEKE, W.; KUGLER, J.O.; SCHOLDERER, J. European consumer acceptance of safetyimproving interventions in the beef chain. Food Cont., v. 22, p. 1776-1784, 2011.

ZHAO, R.; QIAO , J.; CHEN, Y. Influencing factors of consumer willingness-to-buy traceable foods: An analysis of survey data from two Chinese cities. Agri. Agricult. Sci. Proc., v. 1, p. 334-343, 2010. 